\title{
BMJ Open Development of an optimised 1:1 physiotherapy intervention post first-time lumbar discectomy: a mixed-methods study
}

\author{
A Rushton, ${ }^{1}$ L White, ${ }^{2}$ A Heap, ${ }^{2}$ M Calvert, ${ }^{3} \mathrm{~N}$ Heneghan, ${ }^{1} \mathrm{P}$ Goodwin ${ }^{4}$
}

To cite: Rushton A, White $L$, Heap A, et al. Development of an optimised 1:1 physiotherapy intervention post first-time lumbar discectomy:

a mixed-methods study. BMJ Open 2016;6:e009409. doi:10.1136/bmjopen-2015009409

- Prepublication history and additional material is available. To view please visit the journal (http://dx.doi.org/ 10.1136/bmjopen-2015009409).

Received 16 July 2015 Revised 6 January 2016 Accepted 10 February 2016

CrossMark

For numbered affiliations see end of article.

Correspondence to Dr A Rushton; a.b.rushton@bham.ac.uk

\section{ABSTRACT}

Objectives: To develop an optimised 1:1

physiotherapy intervention that reflects best practice, with flexibility to tailor management to individual patients, thereby ensuring patient-centred practice.

Design: Mixed-methods combining evidence synthesis, expert review and focus groups.

Setting: Secondary care involving 5 UK specialist spinal centres.

Participants: A purposive panel of clinical experts from the 5 spinal centres, comprising spinal surgeons, inpatient and outpatient physiotherapists, provided expert review of the draft intervention.

Purposive samples of patients $(n=10)$ and physiotherapists $(n=10)$ (inpatient/outpatient physiotherapists managing patients with lumbar discectomy) were invited to participate in the focus groups at 1 spinal centre.

Methods: A draft intervention developed from 2 systematic reviews; a survey of current practice and research related to stratified care was circulated to the panel of clinical experts. Lead physiotherapists collaborated with physiotherapy and surgeon colleagues to provide feedback that informed the intervention presented at 2 focus groups investigating acceptability to patients and physiotherapists. The focus groups were facilitated by an experienced facilitator, recorded in written and tape-recorded forms by an observer. Tape recordings were transcribed verbatim. Data analysis, conducted by 2 independent researchers, employed an iterative and constant comparative process of (1) initial descriptive coding to identify categories and subsequent themes, and (2) deeper, interpretive coding and thematic analysis enabling concepts to emerge and overarching pattern codes to be identified.

Results: The intervention reflected best available evidence and provided flexibility to ensure patientcentred care. The intervention comprised up to 8 sessions of 1:1 physiotherapy over 8 weeks, starting 4 weeks postsurgery. The intervention was acceptable to patients and physiotherapists.

Conclusions: A rigorous process informed an optimised 1:1 physiotherapy intervention post-lumbar discectomy that reflects best practice. The developed intervention was agreed on by the 5 spinal centres for

\section{Strengths and limitations of this study}

- The study employed a rigorous process to inform development of the 1:1 physiotherapy intervention.

- All stakeholders (physiotherapists, patients and surgeons) were involved in the development process.

- The limitation of this study is its starting point, that there is minimal evidence to support existing physiotherapy interventions post-lumbar discectomy.

- Limited evidence was available, supporting the necessity of including expert opinion to develop the intervention.

- The strengths of the developed intervention are that it is acceptable to patients and clinicians, and is informed by the best available evidence.

implementation in a randomised controlled trial to evaluate its effectiveness.

\section{INTRODUCTION}

Low back pain that affects $80 \%$ of the population within their lifetime ${ }^{1}$ contributes to estimated costs of $£ 10668$ million annually ${ }^{2}$ owing to lost productivity and sickness/disability benefit, although this is now most likely an underestimate. The largest single component of expenditure in managing low back pain is surgery that includes lumbar discectomy to excise part of a prolapsed intervertebral disc for a primary indication of leg pain (radiculopathy). It has been estimated that the direct/indirect costs of managing patients experiencing leg pain originating from disc pressure in the Netherlands are $€ 1.2$ billion annually. ${ }^{3}$ In the year 2013/2014, 8478 lumbar discectomies were performed within the UK National Health Service (NHS). ${ }^{4}$ The most recent 
data estimate 12000 operations per year in the Netherlands ${ }^{5}$ and 287122 in the USA. ${ }^{6}$ Lumbar discectomy is considered an effective surgical procedure, with reported success rates of $46-75 \%$ at $6-8$ weeks, and $78-$ $95 \%$ at $1-2$ years postsurgery, ${ }^{7}$ although varied definitions of success contribute to the variability of defined rates.

The mean age of patients undergoing surgery is 45 years $^{4}$ and most patients are therefore working. The literature suggests that only $70 \%$ are fit to return to work 12 months after surgery, ${ }^{8}$ with $30-70 \%$ experiencing residual pain, ${ }^{9}$ and $3-12 \%$ patients requiring further surgery. ${ }^{10}$ In the UK, in 2013/2014, 1164 (approximately $14 \%)$ revision operations were performed. ${ }^{4}$ Compounding this, the mean hospital stay for first-time lumbar discectomy has reduced considerably from 6.6 $(1999 / 2000)$ to 2.3 days $(2013 / 2014),{ }^{4}$ limiting the opportunity for inpatient postoperative advice and rehabilitation. Outpatient postoperative rehabilitation is therefore a key issue.

In a UK audit of spinal surgeons ${ }^{11}$ and a survey of physiotherapy management post-lumbar discectomy, ${ }^{12}$ postoperative advice and rehabilitation was variable. McGregor et $a l^{11}$ found, for example, that advice to avoid sitting postoperatively ranged from 2 to 42 days, and that 55\% surgeons referred patients routinely for physiotherapy postdischarge. Williamson et $a l^{12}$ found that individualised (1:1) outpatient physiotherapy was provided for all patients routinely in $44 \%$ of spinal centres; and that in a further $46 \%$ centres, patients were seen by physiotherapists only if they were experiencing residual problems. Content and advice of physiotherapy provided was variable; for example, the number of sessions ranged from 1 to 20 , and a wide range of type of exercises were advocated. The surveys highlighted the need for research to optimise rehabilitation for this patient group.

Our systematic review ${ }^{13}$ investigated the effectiveness of all physiotherapy outpatient interventions post first single-level lumbar discectomy. Most trials involved group rehabilitation, with three trials investigating 1:1 physiotherapy management, reflective of current practice $^{12}$ in several countries including the UK. Of the $n=16$ included trials, only 1 was assessed as low risk of bias. Overall, we found inconclusive evidence for the effectiveness of outpatient physiotherapy post first lumbar discectomy. Some evidence suggested that physiotherapy improved disability, with a potential benefit of more intensive intervention; and weak evidence suggested improved movement and physical impairment, all in the short term (12 weeks postsurgery).

A recently updated Cochrane systematic review investigated the effectiveness of all rehabilitation programmes (including physiotherapy) post-lumbar disc surgery. ${ }^{7}$ Of the included trials $(n=22), 10$ were assessed as low risk of bias. Through limited statistical pooling, a potential positive effect of exercise on pain and function was demonstrated, with very low/low quality evidence supporting high-intensity over low-intensity exercise programmes in the short term (12 weeks postsurgery). Other key findings were that rehabilitation programmes starting immediately after surgery were no more effective than control; low/very low quality evidence supported no differences between programmes starting 4-6 weeks after surgery; and low-quality evidence supported physiotherapy starting at 4-6 weeks compared with no treatment or education only. This review updated Ostelo et al s review ${ }^{14}$ that included $\mathrm{n}=14$ trials and had previously found low-to-moderate evidence supporting the effectiveness of exercise as being more effective than no treatment, and high-intensity exercises as being more effective than low-intensity exercises for pain and improved physical impairment.

There is minimal evidence to support existing physiotherapy interventions post-lumbar discectomy. The systematic reviews indicate the need for further evidence. In addition, substantial heterogeneity in treatment effects could be explained by variation in the quality, administration and components of interventions, illustrated by the documented variability in management and advice. ${ }^{11}{ }^{12}$ An adequately powered low risk of bias trial is required to identify whether $1: 1$ physiotherapy is more effective than education alone, and whether it would be a cost-effective use of resources. However, prior to planning a trial, the best practice $1: 1$ physiotherapy intervention required development to provide an optimised intervention in terms of timing, number of treatment sessions and content.

\section{Objective}

To develop an optimised 1:1 physiotherapy intervention that reflects best practice, with flexibility to tailor management to individual patients thereby ensuring patientcentred practice, in line with Medical Research Council (MRC) guidance regarding the development and evaluation of complex interventions. ${ }^{15}$

\section{METHODS \\ Design}

Mixed-methods sequential design combining evidence synthesis, expert review and focus groups.

\section{Process for development of intervention}

The intervention was developed in 2012 and agreed on by the research team, clinical experts and spinal surgeons at five UK specialist spinal centres (planned sites for future trial: University Hospitals Birmingham NHS Foundation Trust, Salford Royal NHS Foundation Trust, Royal Liverpool and Broadgreen University Hospitals NHS Trust, Newcastle upon Tyne Hospitals NHS Foundation Trust, The Robert Jones and Agnes Hunt Orthopaedic and District Hospital NHS Trust), outpatient department physiotherapists, and users (patients and carers) through the following stages. 


\section{Stage 1}

A draft intervention was developed by the research team using the existing evidence ${ }^{15}$ from the two systematic reviews and survey of current practice for lumbar discectomy, and research related to stratified care:

- Ostelo et $a l^{14}$ explored literature to May 2007, investigating active rehabilitation programmes aimed at functional restoration, including supervised exercise therapy, functional restoration programmes and rehabilitation-orientated approaches in rehabilitation medicine. This review excluded interventions solely targeting pain relief, or improvement of physical outcomes of strength or flexibility, that reflect aspects of 1:1 physiotherapy intervention.

- Rushton et $a l^{13}$ explored literature to December 2009 investigating all physiotherapy outpatient management approaches, including 1:1 physiotherapy intervention.

- Williamson et $a l^{12}{ }^{12}$ in a UK survey, explored current practice for managing patients post-lumbar discectomy using a total population sample of physiotherapists working in spinal centres.

- Hill et $a l^{16}$ developed the validated STarT back tool that is intended to screen patients with low back pain in a primary care context, to enable subgrouping of patients regarding their risk of poor outcome (persistent disability).

Through research team discussion (involving all authors), the evidence was synthesised and key research findings (conclusions from included literature in the context of risk of bias to ensure rigour) to inform clinical practice were extracted to produce an initial draft intervention. The draft intervention consisted of underlying principles for the intervention (key findings from systematic reviews and research related to stratified care) and specific content detailing possible physiotherapy treatments (key findings from surveys of current practice). The draft intervention was further discussed and revised by the research team to produce an agreed version for clinical expert review.

\section{Stage 2}

The draft intervention was circulated to the clinical experts (clinical specialist physiotherapists and surgeons with expertise in managing patients pre- and postlumbar discectomy) at five spinal centres for their review and comments. The experts were asked to liaise with their spinal surgeons to discuss the intervention and its potential implementation. The feedback from clinical experts and surgeons was integrated into the proposed intervention through subsequent drafts by the research team.

\section{Stage 3}

Two (patients and physiotherapists) focus groups ${ }^{17}$ took place at one clinical site (University Hospitals Birmingham NHS Foundation Trust), to discuss the draft intervention and its potential for implementation as a framework for clinical decision-making. The key objective of the focus groups was to evaluate acceptability of the evidence-based intervention. Questions to prompt consideration of acceptability included: What do you like about it? Is there anything that you do not like about it? Would you have found it helpful? Can it be improved? Any other thoughts? Participants in the physiotherapist focus group comprised physiotherapists treating patients following lumbar discectomy. Participants in the patient focus group were all users, including patients who had received lumbar discectomy surgery and their carers. Participants were approached by a Trust physiotherapist.

The focus groups started by the agreement of established ground rules and gaining written informed consent. An overview of the proposed physiotherapy intervention was presented and time was given for participants to review the intervention; the intervention was discussed and key points agreed on before summarising the discussion and closing the group. The focus groups were facilitated by an experienced facilitator (AR), recorded in written and tape-recorded forms by an observer (Christine Wright), with a patient on the study team assisting the participants throughout. The tape recordings were transcribed verbatim. Data analysis was initially conducted independently by two researchers $(\mathrm{AR} / \mathrm{CW})$ using an iterative process of initial descriptive coding to identify categories and subsequent themes. Deeper interpretive coding and thematic analysis followed, enabling concepts to emerge and overarching pattern codes to be identified. ${ }^{18}$ The two independent analyses were brought together through discussion of overarching patterns that reconciled any differences. Constant comparison was used throughout the analytic process permitting exploration of deviant/negative experiences. ${ }^{19}$ Rigour was ensured through attention to key issues, for example, potential researcher bias, and the recorder maintaining a summary of the focus group dynamics and therefore any potential influence on participant responses. Amendments were made to the intervention based on these findings.

\section{Stage 4}

The final intervention was circulated through email to the clinical experts at the five spinal centres for any final comments, and their agreement was sought regarding the acceptability of the intervention for implementation as part of a future clinical trial to assess effectiveness of the developed intervention of 1:1 physiotherapy management post first lumbar discectomy.

\section{Participants in the expert review stages}

A purposive panel of clinical experts was selected at five spinal centres to ensure that those with an expert knowledge of the problem were included, that is, spinal surgeons (orthopaedic and neurosurgeons), and inpatient and outpatient physiotherapists with experience in managing patients pre- and post-lumbar discectomy. A letter, 
participant information sheet and questionnaire were sent to the lead physiotherapist at each site. The sites were part of a collaboration planning to participate as sites for the future trial. The lead physiotherapist collaborated with physiotherapy and surgeon colleagues to inform their response. Consent was assumed by way of returned feedback to the researcher by post or email. Non-responders were reminded twice by either email or telephone.

\section{Participant focus groups}

A purposive sample of patients who had experienced lumbar discectomy surgery within the previous 6 months $(n=10)$ was identified at one spinal centre by a Trust physiotherapist and invited to participate in a focus group (purposive for male, female, varied cultural backgrounds and ages across patients). A participant information sheet was forwarded to each patient along with details of the location, timing and purpose of the focus group. A purposive sample of physiotherapists $(n=10$; including inpatient and outpatient physiotherapists managing patients with lumbar discectomy) was invited to participate in the focus group at one spinal centre.

\section{FINDINGS}

\section{Stage 1}

Principles guiding the development of the intervention were informed by the literature. ${ }^{13}{ }^{14}$ The initial draft of the intervention reflected a framework of the best available evidence but also provided flexibility of the intervention to ensure patient-centred practice. Content encompassed the following components: education, advice, mobility exercises, core stability exercises, a progressive approach to exercise and encouragement of early return to work and activity. ${ }^{12}$ It was defined that patients would attend up to eight physiotherapy sessions over a period of up to 8 weeks (to allow for patient choice and variations in practice across sites), starting at approximately 4 weeks postsurgery to provide optimal care. ${ }^{12-14}$ The 1:1 physiotherapy would be delivered in line with current research advocating a targeted approach to treatment basing treatment decisions on data and clinical reasoning regarding the individual patient.

\section{Stage 2}

All participants were in agreement with the principles and content of the intervention. Key changes made at this stage based on feedback were related to the usability of the framework in practice. The formatting was adapted to enable easy completion in busy clinical practice, and a problem list format was employed to structure the record of treatment.

\section{Stage 3}

Patients ( $n=5$ from the $n=10$ invited patients) from one spinal centre participated in the patient focus group.
Inpatient and outpatient physiotherapists $(\mathrm{n}=10)$ currently managing postdiscectomy patients at the same spinal centre participated in the physiotherapist focus group. Changes to the intervention following the focus groups are detailed in boxes 1 and 2. Patients and physiotherapists were broadly in agreement with the intervention, with few suggested additions to the guiding principles and specific content.

\section{Stage 4}

Following circulation to the experts at the five spinal centres, no further changes were proposed and all centres confirmed that the finalised intervention (see online supplementary appendix A) would be acceptable for implementation in practice, in terms of value, completion of the treatment record and timing of delivering the intervention. The evidence-based intervention constitutes an optimised 1:1 physiotherapy intervention with the inherent flexibility to enable physiotherapists to tailor management to individual patients, thereby ensuring patient-centred practice. The intervention consists of underlying principles for the intervention, definition of a problem list and specific physiotherapy treatments linked to potential identified problems.

\section{DISCUSSION}

Physiotherapy management is a complex intervention; that is, it has several interacting components and is tailored to the individual patient through a physiotherapist's clinical reasoning process, and is varied in its design (eg, addressing physical and psychosocial aspects), delivery and local application. ${ }^{15}$ An essential component of reporting a trial of a complex intervention is being able to describe the components of the intervention and to provide a rationale for their inclusion. For the postdiscectomy population, there was a necessity to develop a physiotherapy intervention reflective of best practice in the UK NHS that was based on

Box 1 Changes to physiotherapy intervention following a focus group with patients

- Ensure that instructions clarify that the patient assessment comes first

- Insert a section at the end of the document to summarise 'exactly where the patient is at discharge and what they have been advised to do'

- At last visit, physiotherapist to reinforce that further support would be via a general practitioner (GP)

- Information in the sections from the 'reduced knowledge to enable self-management' and 'pain' would be useful in the booklet (an associated patient leaflet intervention)-linked to timescales/timelines, for example, expectations of flare-ups and how to manage them, taking painkillers, role of GP

- Include information such as how to manage 'drop foot'within physiotherapy aspects 
Box 2 Changes to physiotherapy intervention following focus group with physiotherapists

Review the phrase 'guided discovery'

- In the guiding principles, reword for clarity of 'increasing intensity' and 'progressive' as two distinct points. Integrate footnote into the bullet points

- Add in liaison with surgical team/colleagues as an intervention where most appropriate

- Add in goal setting (general terms) under self-management problem

- Add in goal setting in a positive context to the guiding principles

- Stress form is to document what you HAVE done, NOT what you have to cover

- Emphasise that the list is covering everything you might want to use - but that you are unlikely to do everything

- Put positives in the principles

- Problem list could change over the weeks; might need more than 5; include a sessions number when each problem is inserted in the list and when it is removed

best available evidence and addressed existing variability of practice.

The 1:1 physiotherapy intervention (online supplementary appendix A) has several interacting components, and is divided into two aspects. First, a list of guiding principles provides the basis for and guides the individual physiotherapist's decisions for goal setting, selecting treatment content, and deciding on dose and treatment progression. Most guiding principles were derived from the existing evidence. Patients attend up to eight sessions, over a period of up to 8 weeks (allowing patient choice and variations in practice), starting at approximately 4 weeks postsurgery to provide optimal care. ${ }^{13}{ }^{14}$ Second, a table of dual purpose provides a (1) description of the intervention and (2) structure to enable physiotherapists to record the patient-specific delivered intervention. Individual treatments are informed by the physiotherapist's assessment of the individual patient and their identified problems, for example, pain or reduced range of movement, and were derived from a combination of the existing evidence and the focus groups (boxes 1 and 2). Physiotherapy decision-making is complex and many trial interventions fail to capture and describe these processes. Facilitating physiotherapists to base intervention decisions on their assessment findings enables an individually tailored intervention for each patient to ensure patient-centred care. From the assessment findings, the intervention guides the physiotherapist to formulate a physiotherapy diagnosis, to identify any reasons for exerting caution, and to define a problem list. The identified problem(s) then linked to possible physiotherapy treatment options. The intervention encompasses a range of treatment intervention options that physiotherapists can employ, including education, advice, mobility exercises, core stability exercises, a progressive approach to exercise and encouragement of early return to work and activity. Finally, it incorporates flexibility for physiotherapists to tailor management to individual patients, thereby ensuring patient-centred practice, in line with MRC guidance for complex interventions. ${ }^{15}$

The strengths of the developed intervention are that it is acceptable to patients and clinicians, and is informed by the best available evidence. ${ }^{11-14}$ This will discourage the use of treatments for which there is evidence of no effect. Although developed prior to the recently updated Cochrane review, ${ }^{7}$ the $1: 1$ intervention remains consistent with the best evidence. Acceptability to clinicians is important to ensure that the intervention can be delivered in a busy NHS department.

The strengths of this study are the rigorous process to inform development of the 1:1 physiotherapy intervention, and that all stakeholders (physiotherapists, patients and surgeons) were involved in the development process. The limitation is interestingly its starting point, that there is minimal evidence to support existing physiotherapy interventions post-lumbar discectomy and that limited evidence was therefore available to inform this intervention. This supports the necessity of including expert opinion to develop the intervention.

\section{CONCLUSIONS}

This paper has described a rigorous process to develop an evidence-based physiotherapy intervention postlumbar discectomy surgery that reflects best practice in a UK NHS clinical setting. The 1:1 physiotherapy intervention is being evaluated through an external pilot and feasibility study where a key aim will be to evaluate acceptability of the intervention and its components to patients. The trial was registered with ClinicalTrials.gov (ISRCTN33808269, assigned 10/12/2012).

\section{Author affiliations}

${ }^{1}$ School of Sport, Exercise and Rehabilitation Sciences, College of Life and Environmental Sciences, University of Birmingham, Birmingham, UK ${ }^{2}$ Department of Physiotherapy, University Hospitals Birmingham NHS Foundation Trust, Queen Elizabeth Hospital, Queen Elizabeth Medical Centre, Birmingham, UK

${ }^{3}$ Primary Care Clinical Sciences, Institute of Applied Health Research, College of Medical and Dental Sciences, University of Birmingham, Birmingham, UK ${ }^{4}$ Health Professions Department (Physiotherapy), Manchester Metropolitan University, Manchester, UK

Acknowledgements The authors thank Claire Littleford, patient user, who supported the running of the focus groups and initial stage of data analysis. Christine Wright, who supported study design and was the observer in the focus groups, prior to retirement.

Contributors $A R$ and $P G$ are senior lecturers in physiotherapy and $N H$ is a lecturer in physiotherapy. MC is a professor of outcomes methodology. $\mathrm{AH}$ and $L W$ are clinical specialists and extended scope practitioners working in spinal surgery. AR, PG, NH, AH and LW have long-standing professional interests in the rehabilitation of patients following spinal surgery, and all have a professional focus on musculoskeletal physiotherapy. AR, PG, AH and LW were responsible for the conception of the study. AR, MC, PG and NH were responsible for the design and methodology. AR was responsible for the data analysis. All authors have contributed to the study and have been involved in developing the content of the article. AR wrote the first draft of the paper and 
has worked with all authors reworking content into subsequent drafts. All authors gave final approval of the version to be published. AR is the guarantor.

Funding Queen Elizabeth Hospital Birmingham Charity, Birmingham, UK to support the costs of the focus groups (project 17-3-780).

Competing interests None declared.

Ethics approval Ethical approval was obtained from the lead author's institution (ERN_10-0474), as this developmental work constitutes therapy evaluation, not necessitating National Research Ethics Service approval as agreed on by the local Research Governance office.

Provenance and peer review Not commissioned; externally peer reviewed.

Data sharing statement No additional data are available.

Open Access This is an Open Access article distributed in accordance with the Creative Commons Attribution Non Commercial (CC BY-NC 4.0) license which permits others to distribute, remix, adapt, build upon this work noncommercially, and license their derivative works on different terms, provided the original work is properly cited and the use is non-commercial. See: http:// creativecommons.org/licenses/by-nc/4.0/

\section{REFERENCES}

1. WHO. WHO Technical Report Series. The burden of musculoskeletal conditions at the start of the new millennium. Geneva: World Health Organisation, 2003.

2. Maniadakis N, Gray A. The economic burden of low back pain in the UK. Pain 2000;84:95-103.

3. HCN. Health Council of the Netherlands. Management of the lumbosacral radicular syndrome (sciatica). The Hague: Health Council of the Netherlands, 1999. Publication no. 1999/18.

4. HES online. All procedures and interventions 2013/14. 3 character All procedures [V33]. http://www.hscic.gov.uk/hes (accessed 3 Mar 2015).
5. van Beek E, Lemmens K, van Schooten G, et al. Reduceren van praktijkvariatie: budgettaire effecten van scherpere indicatiestelling. Reduceren van praktijkvariatie: budgettaire effecten van scherpere indicatiestelling. Breukelen: Plexus, 2010.

6. Sherman J, Cauthen J, Schoenberg D, et al. Economic impact of improving outcomes of lumbar discectomy. Spine J 2010;10:108-16.

7. Oosterhuis T, Costa LO, Maher CG, et al. Rehabilitation after lumbar disc surgery. Cochrane Database Syst Rev 2014;3:CD003007.

8. Donceel P, Du Bois M. Predictors for work incapacity continuing after disc surgery. Scand J Work Health Environ 1998;25:264-71.

9. Ostelo RW, Goossens ME, de Vet HC, et al. Economic evaluation of behavioural-graded activity program compared to physical therapy for patients following lumbar disc surgery. Spine 2004;29:615-22.

10. CBO. The lumbosacral radicular syndrome, Dutch Institute for Healthcare Improvement. Centraal Begeleidings Orgaan Report, 2008.

11. McGregor AH, Dicken B, Jamrozik K. National audit of post-operative management in spinal surgery. BMC Musculoskelet Disord 2006;7:47.

12. Williamson E, White L, Rushton A. A survey of post-operative management for patients following first time lumbar discectomy. Eur Spine J 2007;16:795-802.

13. Rushton A, Wright C, Goodwin $\mathrm{P}$, et al. Physiotherapy rehabilitation post first lumbar discectomy: a systematic review and meta-analysis of randomised controlled trials. Spine 2011;36:E961-72.

14. Ostelo RW, Costa LO, Maher CG, et al. Rehabilitation after lumbar disc surgery. Cochrane Database Syst Rev 2008;(4):CD003007.

15. Craig P, Dieppe P, Macintyre S, et al. Developing and evaluating complex interventions: the new Medical Research Council guidance. BMJ 2008;337:a1655

16. Hill JC, Dunn KM, Lewis $\mathrm{M}$, et al. A primary care back pain screening tool: identifying patient subgroups for initial treatment. Arthritis Rheum 2008;59:632-41.

17. Kitzinger J. Chapter 3 focus groups. In: Pope C, Mays N, eds. Qualitative research in health care. Blackwell Publishing. BMJ Books, 2006:21-31.

18. Miles M, Huberman A. Qualitative data analysis. London: Sage Publications, 1994.

19. Corbin J, Strauss A. Basics of qualitative research. 3rd edn. London: Sage, 2008. 